This item was submitted to Loughborough's Research Repository by the author.

Items in Figshare are protected by copyright, with all rights reserved, unless otherwise indicated.

\title{
Shape waves in 2D Josephson junctions: exact solutions and time dilation
}

PLEASE CITE THE PUBLISHED VERSION

http://dx.doi.org/10.1103/PhysRevLett.101.127002

\section{PUBLISHER}

(C) American Physical Society

VERSION

VoR (Version of Record)

LICENCE

CC BY-NC-ND 4.0

REPOSITORY RECORD

Gulevich, Dmitry R., F.V. Kusmartsev, Sergey Savel'ev, V.A. Yampol'skii, and Franco Nori. 2019. "Shape Waves in 2D Josephson Junctions: Exact Solutions and Time Dilation". figshare.

https://hdl.handle.net/2134/12763. 


\title{
Shape Waves in 2D Josephson Junctions: Exact Solutions and Time Dilation
}

\author{
D. R. Gulevich, ${ }^{1,2}$ F. V. Kusmartsev, ${ }^{1}$ Sergey Savel'ev, ${ }^{1,2}$ V. A. Yampol'skii, ${ }^{2,3}$ and Franco Nori ${ }^{2,4}$ \\ ${ }^{1}$ Physics Department, Loughborough University, Leicestershire, LE11 3TU, United Kingdom \\ ${ }^{2}$ Advanced Science Institute, The Institute of Physical and Chemical Research (RIKEN), Wako-shi, Saitama, 351-0198, Japan \\ ${ }^{3}$ Usikov Institute for Radiophysics and Electronics, Ukrainian Academy of Science, 61085 Kharkov, Ukraine \\ ${ }^{4}$ MCTP, Department of Physics, University of Michigan, Ann Arbor, Michigan 48109, USA
}

(Received 27 April 2008; published 18 September 2008)

\begin{abstract}
We predict a new class of excitations propagating along a Josephson vortex in two-dimensional Josephson junctions. These excitations are associated with the distortion of a Josephson vortex line and have an analogy with shear waves in solid mechanics. Their shapes can have an arbitrary profile, which is retained when propagating. We derive a universal analytical expression for the energy of arbitrary shape excitations, investigate their influence on the dynamics of a vortex line, and discuss conditions where such excitations can be created. Finally, we show that such excitations play the role of a clock for a relativistically moving Josephson vortex and suggest an experiment to measure a time dilation effect analogous to that in special relativity.
\end{abstract}

DOI: 10.1103/PhysRevLett.101.127002

PACS numbers: 74.50.+r, 03.75.Lm, 05.45.Yv

Introduction.-The sine-Gordon (SG) model describes a variety of physical systems, including ferromagnets, information transport in microtubules, dislocations in crystals, nonlinear optics, Josephson junctions, and high temperature superconductors (see, e.g., [1-4]). The $(1+1)$ D SG model is fully integrable and possesses well-known exact solutions in the form of solitons and breathers [3,4]. Depending on the physical realization, they describe different objects, for instance, magnetic domain walls in ferromagnets or vortices (fluxons) in Josephson junctions. There exist mathematical techniques that allow constructing solutions of the $(1+1) \mathrm{D}$ SG, such as the Bäcklund transformation, the Hirota and inverse scattering methods (see, e.g., [3,4]). Although real systems described by the SG model may contain additional terms associated with damping or external conditions, such terms can be taken into account as perturbations to the exact solutions $[5,6]$.

To describe realistic systems, such as two-dimensional Josephson junctions, one should depart from the well studied $(1+1) \mathrm{D}$ model and deal with the $(2+1) \mathrm{D}$ SG equation, which is no longer fully integrable. The extra dimension opens new avenues for the existence of phenomena which are absent in the 1D case (see, e.g., [7-10]). Here we show that there exists a class of exact solutions of the $(2+1) D S G$ equation which describes the propagation of distortions of an arbitrary shape along a Josephson vortex line. The property of these excitations such as transmitting pulses of electromagnetic radiation of arbitrary shape along a Josephson vortex can be useful for the transmission of information in various Josephson devices (see, e.g., theoretical and experimental works [11,12]).

Experiments [13] have shown that Josephson vortices can exhibit Lorentz contraction. So far, another relativistic effect [14], time dilation, has been considered difficult to detect in Josephson systems. In order to observe time dilation, an internal degree of freedom acting as a clock is needed. In $(1+1) \mathrm{D}$ such clock is absent because solitons of the $(1+1) \mathrm{D} S \mathrm{SG}$ equation do not possess internal oscillation modes. Recently, time dilation was proposed [15] in junctions obeying the double sine-Gordon equation. There, an internal degree of freedom of a vortex, due to a special feature of the double SG model was proposed as a clock for a moving vortex. Here we show that there is no need to deviate from the original pure sine-Gordon nonlinearity because an additional degree of freedom due to an extra dimension, can be used to realize a clock.

Model.-Consider a 2D Josephson junction described by the $(2+1) \mathrm{D} S \mathrm{SG}$ equation,

$$
\varphi_{t t}-\varphi_{x x}-\varphi_{y y}+\sin \varphi=0
$$

where $\varphi$ is the superconducting phase difference across the Josephson junction. Here, the coordinates $x$ and $y$ are normalized by the Josephson penetration length $\lambda_{J}$, and the time $t$ is normalized by the inverse Josephson plasma frequency $\omega_{p}^{-1}$ [2]. In the case of a Josephson junction, the soliton solutions of Eq. (1) describe Josephson vortices or fluxons [2-5].

A more general class of solutions of the $(2+1)$ D SG equation has the following form,

$$
\varphi(x, y, t)=4 \arctan \exp [y-f(x \pm t)],
$$

which satisfies Eq. (1) exactly with an arbitrary real-valued twice-differentiable function $f(\xi)$. The excitations, described by $f$, are associated with a local shift of a Josephson vortex in the transverse direction and have analogies with elastic shear waves in, e.g., solid mechanics and seismology [16]. Such distortions propagate along the stationary vortex line with the fixed speed $|u|=1$ in units of the Swihart velocity $\bar{c}=\lambda_{J} \omega_{p}$. Hereafter, we will refer to these excitations as shape waves.

Because of the arbitrariness of $f$, Eq. (2) describes a variety of excitations of various shapes. Choosing $f$ local- 
ized in a finite area, e.g., $f=A / \cosh (x-t)$, Eq. (2) describes an excitation, localized along $x$ that keeps its shape when propagating, i.e., a solitary wave [4]. For each solitary wave of this type, there exists an antipartner with an $f$ of opposite sign in Eq. (2). For solitary waves to be solitons, there is an additional important criterion: restoring their shapes after they collide. Consider a trial function $\varphi(x, y, t)=4 \arctan \exp [y-f(x+t) \pm f(x-t)] \quad$ that, when $t \rightarrow-\infty$, describes the propagation of two solitary shape waves toward each other (minus sign) or a solitary wave and its antipartner (plus sign). One can see that (2) can only approximately satisfy Eq. (1) when $\mid f^{\prime}(x+$ t) $f^{\prime}(x-t) \mid \ll 1$ for all values of $x$ and $t$. This suggests that, in general, the condition for restoring the shapes may not be satisfied. Indeed, our numerical solutions of the $(2+$ 1)D SG equation show that the waves keep their shapes when propagating, but their collision is destructive. The collision of two waves of large amplitudes may lead to their mutual annihilation, dissipating energy away from the vortex; see Fig. 1. However, smaller-amplitude waves behave similarly to solitons, dissipating very small energy and keeping their original shapes after collision.

Since the Eq. (1) is Lorentz invariant, one can obtain other solutions performing Lorentz transformations on Eq. (2). A Lorentz transformation along the $x$ axis leads to a Lorentz contraction of the excitation, but does not lead to any solution outside the class of Eq. (2), only rescaling the arbitrary function $f(\xi)$. The Lorentz transformation

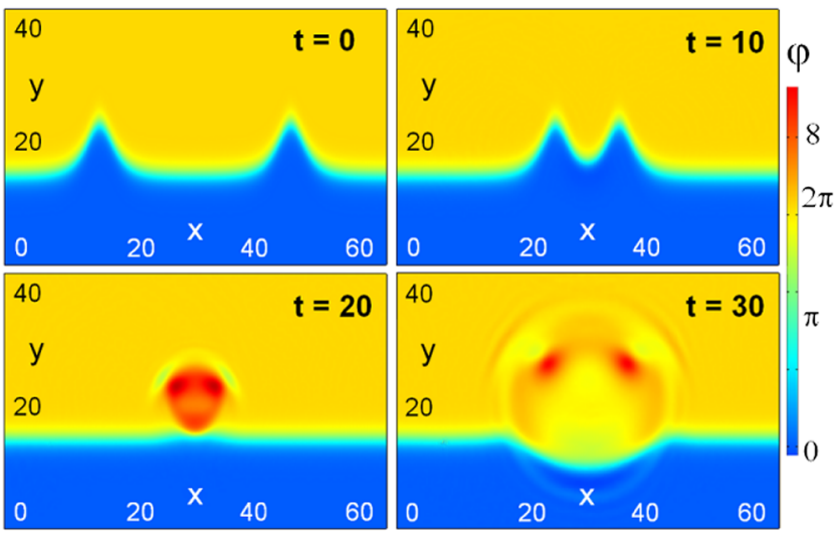

FIG. 1 (color online). Collision and annihilation of two largeamplitude solitary shape waves propagating along a Josephson vortex line, Eq. (2) with $f=8 / \cosh \left[\left(x-x_{0} \pm t\right) / 2\right]$. The color scale represents the superconducting phase difference $\varphi$. The figure shows $\varphi(x, y, t)$ snapshots obtained by numerically solving the $(2+1)$ D SG equation on a region of size $60 \times 40 \lambda_{J}^{2}$ with Neumann boundary conditions: $\mathbf{n} \cdot \nabla \varphi=0$. Initially, the two solitary waves, moving at speed $|u|=1$ toward each other, keep their shape while propagating, but their collision at $t \sim 15$ leads to their mutual annihilation and radiation of energy in all directions. Contrary to the large-amplitude waves shown here, small-amplitude shape waves satisfying $\left|f^{\prime}(x+t) f^{\prime}(x-t)\right| \ll$ 1 , interact weakly during the collision and behave similarly to solitons. along $y$ turns out to be far more interesting, leading to a class of solutions more general than Eq. (2). After such transformation,

$$
\varphi(x, y, t)=4 \arctan \exp \left[\frac{y-v t}{\sqrt{1-v^{2}}}-f\left(x \pm \frac{t-v y}{\sqrt{1-v^{2}}}\right)\right]
$$

which describes a Josephson vortex line moving with $-1<v<1$ and a propagating shape excitation described by $f(\xi)$.

We will show that, apart from the Lorentz contraction [13], a propagating Josephson vortex exhibits time dilation which influences the dynamics of its shape excitations, Eq. (3). Recently, the Lorentz time dilation of a bound half-fluxon pair has been numerically studied [15] for a long Josephson junction with a ferromagnetic insulator. The use of the internal degree of freedom of a bound half-fluxon pair was proposed there to test the time dilation. It was found that internal oscillations of the bound pair can play the role of a clock [15]. A Lorentz reduction of their frequency was numerically observed [15] when the bound half-fluxon pair was moving with relativistic velocities. Here we demonstrate that, to observe the time dilation effect there is no need to use the double sine-Gordon equation [15] but the additional degree of freedom of the $(2+1) \mathrm{D}$ SG equation can be used to realize the internal clock. Indeed, the shape excitation moving along a fluxon provides such a clock. In other words, the position of the excitation on a fluxon acts as a "minute hand." From Eq. (3) the position of a vortex changes with time as $y_{0}(t)=v t$, while the position of the excitation is $x_{0}(t)=$ $\mp\left[t-v y_{0}(t)\right] /\left(1-v^{2}\right)^{1 / 2}=\mp t\left(1-v^{2}\right)^{1 / 2}$. Thus, the excitation moves along $x$ with speed $|u|=\left|\dot{x}_{0}\right|=$ $\left(1-v^{2}\right)^{1 / 2}<1$. In other words, all dynamic processes related to a relativistic moving vortex are slowed down by a time dilation factor $\left(1-v^{2}\right)^{1 / 2}$, as in special relativity, compared to the stationary vortex. Our numerical calculations confirm that the collision of two solitary waves is also slowed down, compared to the collision at the rest frame as in Fig. 1.

Experimentally, shape waves can be excited when a fluxon line interacts with inhomogeneities introduced on its path. When a vortex line passes through a shaped region of a Josephson junction, shape distortions of a vortex line are excited by inhomogeneities of the boundary. The shape of the excited waves is determined by the shape of the inhomogeneity as well as the vortex velocity. Below we present our analytical and numerical results for the case of a vortex line interacting with a barrier formed by a Josephson junction with a locally-increased width, as shown in Fig. 2(a). Another intriguing possibility to generate the shape excitation is to use a $T$ junction to create a cloning barrier [9] on the path of a propagating fluxon line. Other types of inhomogeneities, besides the geometrical ones, such as microshorts or microresistors, realized by 


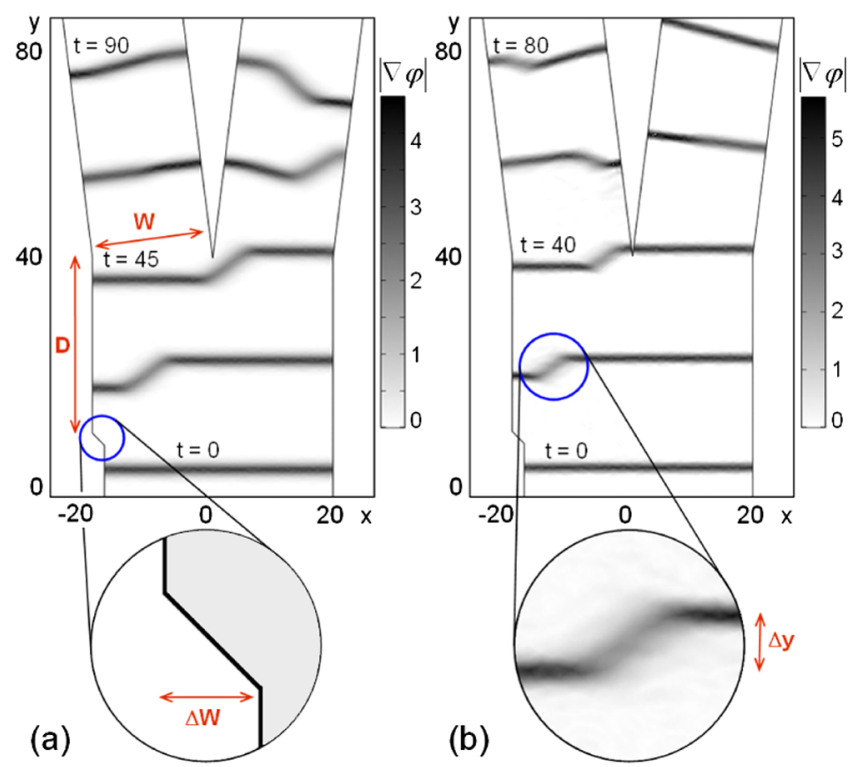

FIG. 2 (color online). Detection of a vortex shape excitation and measurement of its speed in a $Y$ junction. The figure shows snapshots of the superconducting phase gradient $|\nabla \varphi|$ obtained by numerically solving the $(2+1) \mathrm{D}$ SG equation in a $Y$ junction geometry of size $40 \times 80 \lambda_{J}^{2}$ with Neumann boundary conditions: $\mathbf{n} \cdot \nabla \varphi=0$. At time $t=0$, a fluxon line moving with velocity $v=0.8$ (a) or $v=0.9$ (b) approaches a geometrical energy barrier (shown on the left inset). When passing the barrier, a shape excitation (zoomed on the right inset) is induced on the left end of a fluxon line and propagating to the right along the vortex. The propagating fluxon is split by the $Y$ junction in two parts, one containing the shape excitation, and the part without it. Depending on velocity $v$ of the vortex line, the shape excitation can be directed either to the right branch, if the velocity $v$ is low (a), or, to the left branch, if $v$ is higher (b). This is due to the time dilation reducting the speed of the shape excitation. Measuring the critical velocity $v_{c}$ of the vortex line when such a transition occurs, the speed of a shape excitation can be found as $u=$ $v_{c} W / D$. The presence of a shape excitation reduces the average velocity $\langle v\rangle$ of a vortex line, for instance, in (b) the vortex in the left branch at $t=80$ is noticeably delayed from the vortex in the right branch of the $Y$ junction.

variation of the critical current (see, e.g., [5]) can initiate shape excitations. One more possibility is using injectors of electric current connected locally to a Josephson junction [17] where the fluxon line is propagating. This allows to conveniently control the shape of the excitations by varying the electric current of the injectors.

In order to detect the propagation of a shape wave we propose the following scheme which involves the use of a $Y$-shaped cloning junction [9]. Such junction can split a vortex into two parts, which afterward propagate independently from each other. For this purpose, a $Y$ junction with a small span angle is preferable, in order to minimize the disturbance on the vortex line. When a vortex with a propagating shape wave is split by such a junction, the shape excitation is directed to one of the branches of the $Y$ junction; see Fig. 2. A shape wave can be generated from a boundary by locally increasing the width of the Josephson junction, as shown in the inset of Fig. 2(a). Because of the associated energy barrier, one end of the fluxon line is delayed with respect to the other one, which results in a kink profile, as shown in Fig. 2. The generated kink starts to propagate along the vortex line away from the boundary, carrying information about the induced delay to the rest of the vortex.

Consider now an arbitrary shape excitation with a function $f$ satisfying $f(-W) \rightarrow-\Delta f / 2, f(W) \rightarrow \Delta f / 2$ for $W \rightarrow \infty$. The total energy of this shape excitation is the difference of the energy $E[\varphi]$ of the solution Eq. (3) and the energy $E[\varphi]_{f \equiv 0}$ of the vortex without excitation: $\Delta E=$ $E[\varphi]-E[\varphi]_{f=0}$. We obtain the following analytic expression for the total energy:

$$
\Delta E=\frac{8}{\sqrt{1-v^{2}}}\left[\int_{-\infty}^{\infty} f^{\prime}(x)^{2} d x-v \Delta f\right]
$$

which is exact in the limit $W \rightarrow \infty$ and valid for an arbitrary excitation $f(\xi)$ of height $\Delta f$. As seen from Eq. (4), the energy $\Delta E$ can take negative values if $f$ is a kink $(\Delta f \neq$ 0 ) propagating along a moving vortex line. Indeed, this is in agreement with our numerical simulations on Fig. 2 which indicate that the increased width $\Delta W$ of the Josephson junction is compensated by the energy gained due to the propagating kink with negative $\Delta E$. Neglecting radiation losses, we now write the conservation of energy relation for a moving vortex line: $\Delta E+8 \Delta W(1-$ $\left.v^{2}\right)^{-1 / 2}=0$. Using Eq. (4), the minimal size of the kink can be estimated: $\Delta f \geq \Delta f_{\min }(\Delta W, v)=\Delta W / v$. Note that the apparent height $\Delta y$ of a kink propagating along a moving vortex line is Lorentz contracted: $\Delta y=$ $\Delta f \sqrt{1-v^{2}}$. To describe the kinks in Fig. 2 we use the approximation $f(\xi)=\frac{1}{2} \Delta f \tanh (2 v \xi / \Delta f)$. Substituting it in Eq. (4) we obtain $\Delta E=-8 \Delta f v\left(1-v^{2}\right)^{-1 / 2} / 3$. From energy conservation, we calculate the apparent height of the kink to be $\Delta y=3 \sqrt{1-v^{2}} \Delta W / v$. Substituting the value $\Delta W=2$, we obtain the following results for the apparent heights of the kinks: $\Delta y \simeq 4.5$ for $v=0.8$ and $\Delta y \simeq 2.9$ for $v=0.9$, which agree with the numerical results shown in Figs. 2(a) and 2(b) respectively.

After passing the $Y$ junction, each half of the initial vortex line is directed to a Josephson junction of width $W$. The presence of the shape kink slows down the average speed of a vortex line,

$$
\langle v\rangle=-\frac{1}{2 \pi W} \iint \dot{\varphi} d x d y=v-\frac{\Delta y \sqrt{1-v^{2}}}{W} .
$$

Thus, the presence of a shape wave can be directly detected by measuring the voltage on the Josephson junction, proportional to $\langle v\rangle$. A convenient system to study shape excitations would be a $Y$ junction introduced into a ring, forming a $\sigma$-shaped Josephson junction which has been 
recently realized experimentally with a high- $T_{c}$ superconductor in the context of generation of Terahertz waves [10].

The effect of time dilation is to slow down the shape excitation when the vortex line reaches relativistic velocities. If the velocity of the shape excitation is smaller than some critical value, it will be directed to the left branch of the $Y$ junction, as on the Fig. 2(b), while the vortex propagating in the right branch will remain undisturbed. On the other hand, if the shape excitation propagates faster, it will be directed to the right branch, Fig. 2(a). Because of the change in the average velocity of a vortex line Eq. (5), a voltage step, corresponding to a shape wave changing the branch of the $Y$ junction, will be exhibited on the $I-V$ characteristics. Finding the velocity of a fluxon line $v_{c}$ from the position of the voltage step, speed of the shape excitation can be calculated as $u=v_{c} W / D$, where $D$ is a distance between the barrier and the $Y$ junction; see Fig. 2. In this way, the relativistic factor $\left(1-v^{2}\right)^{1 / 2}$ for the delay of the shape kink propagation can be tested.

In an underdamped Josephson junction, a fluxon can be accelerated to velocities close to the Swihart velocity, typically of the order of $10^{7} \mathrm{~m} / \mathrm{s}^{2}$ for Nb Josephson junctions. The velocity of a fluxon depends both on damping and an applied current [5]. For the damping parameter $\alpha=$ 0.01 and current $\gamma=0.03$ in normalized units [5], it is possible to accelerate a vortex to the velocity $v \simeq 0.92$, so that the relativistic time dilation factor would be $(1-$ $\left.v^{2}\right)^{1 / 2} \simeq 0.4$. For instance, for typical $\mathrm{Nb} / \mathrm{Al}-\mathrm{AlO}_{x} / \mathrm{Nb}$ Josephson junctions with the plasma frequency $\omega_{p} / 2 \pi \sim$ $50 \mathrm{GHz}$, the propagation time of the shape excitation between the two ends of a vortex line in a configuration as in Fig. 2 would be approximately 0.4 ns for a stationary vortex, while for a vortex moving with velocity $v=0.92$ this value would be relativistically delayed by $0.6 \mathrm{~ns}$.

Conclusion.-We have predicted the existence of excitations of arbitrary shapes propagating along stationary or moving Josephson vortices in 2D Josephson junctions, and presented the exact solutions of the $(2+1) \mathrm{D} \mathrm{SG}$ equation. Remarkably, at some conditions, a shape wave can carry negative energy; i.e., a vortex with a shape excitation can have smaller energy than the same vortex without it. Based on our analysis, we suggest how to test a time dilation effect analogous to that in special relativity: using the shape excitation as a minute hand measuring the proper time of the vortex's coordinate frame.

We acknowledge partial support from the NSA, LPS, ARO, NSF Grant No. EIA-0130383, JSPS-RFBR No. 0602-91200, JSPC-CTC program, and MEXT Grant-in-Aid No. 18740224. Also, support from EPSRC via No. EP/ D072581/1, EP/E042589/1, EP/F005482/1, and ESF network program "AQDJJ."
[1] D. L. Mills, Nonlinear Optics (Springer, Berlin, 1998); Yu. S. Kivshar and B. A. Malomed, Rev. Mod. Phys. 61, 763 (1989)

[2] A. Barone and G. Paternó, Physics and Applications of Josephson Effect (Wiley, New York, 1982); Nonlinear Superconducting Devices and High-Tc Materials edited by R. D. Parmentier and N. F. Pedersen (World Scientific, Singapore, 1995).

[3] P. G. Drazin and R. S. Johnson, Solitons: An Introduction (Cambridge University Press, Cambridge, England, 1989); E. A. Jackson, Perspectives in Nonlinear Dynamics (Cambridge University Press, Cambridge, England, 1990).

[4] R. Rajaraman, Solitons and Instantons (Elsevier, Amsterdam, 1982).

[5] D. W. McLaughlin and A. C. Scott, Phys. Rev. A 18, 1652 (1978).

[6] D. R. Gulevich and F. V. Kusmartsev, Phys. Rev. B 74, 214303 (2006).

[7] I. L. Bogolyubskii and V. G. Makhan'kov, JETP Lett. 24, 12 (1976); 25107 (1977); P.L. Christiansen and O. H. Olsen, Phys. Lett. A 68, 185 (1978); P. L. Christiansen and P. S. Lomdahl, Physica (Amsterdam) 2D, 482 (1981); B. Piette and W. J. Zakrzewski, Nonlinearity 11, 1103 (1998); P. L. Christiansen et al., Phys. Scr. 55, 131 (1997).

[8] S. G. Lachenmann et al., Phys. Rev. B 48, 16623 (1993); J. G. Caputo et al., Phys. Rev. E 54, 2092 (1996); C. Gorria et al., Phys. Rev. B 69, 134506 (2004).

[9] D. R. Gulevich and F. V. Kusmartsev, Phys. Rev. Lett. 97, 017004 (2006); Supercond. Sci. Technol. 20, S60 (2007); New J. Phys. 9, 59 (2007).

[10] D. R. Gulevich, M. Gaifullin, O.E. Kusmartseva, F. V. Kusmartsev, and K. Hirata, Physica (Amsterdam) C468, 1903 (2008).

[11] S. Savel'ev, A. Rakhmanov, and F. Nori, Phys. Rev. Lett. 94, 157004 (2005); 98, 077002 (2007); S. Savel'ev, V. Yampol'skii, and F. Nori, ibid. 95, 187002 (2005); S. Savel'ev, A. Rakhmanov, V. Yampol'skii, and F. Nori, Nature Phys. 2, 521 (2006).

[12] V.P. Koshelets and S. V. Shitov, Supercond. Sci. Technol. 13, R53 (2000); V.P. Koshelets et al., IEEE Trans. Appl. Supercond. 17, 336 (2007); M. Yu. Torgashin et al., ibid. 17, 379 (2007).

[13] A. Matsuda and T. Kawakami, Phys. Rev. Lett. 51, 694 (1983); A. Laub et al., ibid. 75, 1372 (1995).

[14] Note that the relativistic dynamics of Josephson junctions is defined by the Swihart velocity $\bar{c}$ which plays a similar role to the speed of light in special relativity.

[15] M. Nishida, K. Murata, T. Fujii, and N. Hatakenaka, Phys. Rev. Lett. 99, 207004 (2007).

[16] Y.C. Fung, Foundations of Solid Mechanics (Prentice Hall, New Jersey, 1965); C. H. Scholz, The Mechanics of Earthquakes and Faulting (Cambridge University Press, Cambridge, England, 2002).

[17] A. V. Ustinov, Appl. Phys. Lett. 80, 3153 (2002); B. A. Malomed and A. V. Ustinov, Phys. Rev. B 69, 064502 (2004). 